\title{
THE SECOND MEAN VALUE THEOREM FOR SUMMABLE FUNCTIONS *
}

\author{
BY M. B. PORTER
}

We start with the following lemma: If $f(x)$ is summable over the interval $(a, b)$ and hence has an indefinite integral $F(x)$ over $(a, x), a \leqq x \leqq b$, then

$$
\begin{aligned}
\lim _{h \rightarrow 0} \int_{a}^{b} \frac{f(x+h)-f(x)}{h} d x & =\lim _{h \rightarrow 0} \frac{F(b+h)-F(b)}{h} \\
- & \lim _{h \rightarrow 0} \frac{F(a+h)-F(a)}{h}=f(b)-f(a),
\end{aligned}
$$

provided that $f(x)$ is right (or left) continuous at the ends of the interval.

Now let $\phi(x)$ denote a monotone function right (or left) continuous at $a$ and $b$, and consider the identity

$$
\int_{a}^{b} \frac{F(x+h) \phi(x+h)-F(x) \phi(x)}{h} d x=(\mathrm{I})+(\mathrm{II}),
$$

where

$$
\begin{aligned}
(\mathrm{I}) & =\int_{a}^{b} \frac{F(x+h)-F(x)}{h} \phi(x+h) d x, \\
\text { (II) } & =\int_{a}^{b} F(x) \frac{\phi(x+h)-\phi(x)}{h} d x .
\end{aligned}
$$

Applying a well known theorem of Lebesgue's to (I), and the first mean value theorem to (II), since we know that the expression $[\phi(x+h)-\phi(x)] / h$ is always of the same sign when $a \leqq x \leqq b$, we have

$$
\begin{aligned}
F(b) \phi(b)-F(a) \phi(a)+\epsilon_{h}{ }^{\prime} & =\int_{a}^{b} f(x) \phi(x) d x \\
& +F\left(\xi_{h}\right) \int_{a}^{b} \frac{\phi(x+h)-\phi(x)}{h} d x+\epsilon_{h}{ }^{\prime \prime}
\end{aligned}
$$

* Presented to the Society, September 18, 1923. 
where $\epsilon_{h}{ }^{\prime}$ and $\epsilon_{h}{ }^{\prime \prime}$ vanish with $h$. Applying the lemma to the last integral, we have:

$$
\begin{array}{rl}
F(b) \phi(b)-F(a) \phi(a)=\int_{a}^{b} & f(x) \phi(x) d x \\
& +F\left(\xi_{h}\right)(\phi(b)-\phi(a))+\epsilon_{h}
\end{array}
$$

where $\lim _{h \rightarrow 0} \epsilon_{h}=0$.

This can be written in the form

$$
\int_{a}^{b} f(x) \phi(x)=\phi(b) \int_{a}^{\xi_{h}} f(x)+\phi(a) \int_{\xi_{h}}^{b} f(x)+\epsilon_{h} .
$$

When $\epsilon_{h}$ takes on the value zero, $F\left(\xi_{h}\right)$, which is continuous, will take on for some value $\xi$, lying between $a$ and $b$, a value such that

$$
\int_{a}^{\infty b} f(x) \phi(x)=\phi(b) \int_{a}^{\xi} f(x)+\phi(a) \int_{\xi}^{b} f(x),
$$

which is Weierstrass's form of the second mean value theorem for integrals.

If, for example, we suppose $\phi(x)$ increasing (monotonically) and replace $\phi(x)$ by $A$ (fixed) over the interval $(a, a+k)$ and by $B$ (fixed) over the interval $(b, b+k)$, leaving the values of $\phi(x)$ unchanged over $(a+k, b)$, we can prove in the same way that

where

$$
\int_{a}^{b} f(x) \phi(x)=A \int_{a}^{\xi} f(x)+B \int_{\xi}^{b} f(x),
$$

$$
A \leqq \phi(x) \leqq B
$$

over $(a, b)$, by applying the same reasoning and letting the parameter $k$ approach zero.

From this form the usual Bonnet forms of the theorem are at once deducible.

The University of Texas 